\title{
Clinical benefit of graduated compression stockings for prevention of venous thromboembolism after total knee arthroplasty: post hoc analysis of a phase 3 clinical study of edoxaban
}

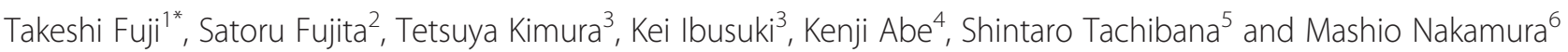

\begin{abstract}
Background: Guidelines from the Japanese Circulation Society recommend prophylaxis with anticoagulation plus intermittent pneumatic compression or graduated compression stockings (GCS) among patients at the highest risk for developing venous thromboembolism (VTE). However, the benefits of concomitant GCS use for patients undergoing total knee arthroplasty (TKA) and receiving anticoagulation remain unknown. In this study, the efficacy of GCS plus anticoagulation compared with anticoagulation alone was evaluated among patients undergoing TKA.
\end{abstract}

Methods: This study is a post hoc analysis of a previously reported phase 3 trial involving patients undergoing TKA. In the primary study, which permitted the use of GCS for mechanical prophylaxis, patients were randomized to receive edoxaban $30 \mathrm{mg}$ once daily or enoxaparin $20 \mathrm{mg}$ twice daily for 11 to 14 days following TKA. The primary endpoint was the incidence of VTE, a composite of symptomatic deep vein thrombosis (DVT), symptomatic pulmonary embolism (PE), and asymptomatic DVT. Treatment comparisons were performed using the chi-square test, and the $95 \%$ confidence intervals were calculated.

Results: Among patients receiving edoxaban, the incidence of VTE was 3.8 and $5.8 \%$ for patients with and without GCS, respectively. For patients receiving enoxaparin, VTE incidence was 8.4 and $20.8 \%$ among those with and without GCS, respectively. Overall, VTE incidence was 6.0 and $13.0 \%$ for anticoagulated patients with and without GCS mechanical prophylaxis, respectively. No deaths or symptomatic PE were reported during this study.

Conclusions: Although the incidence of VTE was >2-fold lower among patients receiving anticoagulation plus GCS compared with those receiving anticoagulation alone, statistical significance was not achieved. Further studies are required to confirm the findings of this preliminary analysis.

Trial registration: ClinicalTrials.gov Identifier: NCT01181102

Keywords: Venous thromboembolism, Deep vein thrombosis, Intermittent pneumatic compression, Graduated compression stockings, Edoxaban, Enoxaparin, Total knee arthroplasty, Orthopedic surgery, VTE, DVT

\footnotetext{
* Correspondence: fuji-th@umin.ac.jp

${ }^{1}$ Department of Orthopedic Surgery, Japan Community Healthcare

Organization Osaka Hospital, 4-2-78 Fukushima, Fukushima-ku, Osaka

553-0003, Japan

Full list of author information is available at the end of the article
} 


\section{Background}

Major lower limb orthopedic surgery-such as total knee arthroplasty (TKA), total hip arthroplasty (THA), and hip fracture surgery (HFS) - is a significant risk factor for postoperative venous thromboembolism (VTE), encompassing deep vein thrombosis (DVT) and pulmonary embolism (PE) [1, 2]. For patients undergoing lower limb orthopedic surgery, several pharmacological and mechanical therapies are available for the prevention of VTE, including graduated compression stockings (GCS), which are a convenient and inexpensive mechanical prophylaxis measure [1]. Although the exact mechanism of action of GCS remains unclear, they are believed to reduce the total cross-sectional area of the leg veins, thereby increasing venous blood flow velocity and preventing venous stasis in the lower limbs [3].

Guidelines from the Japanese Circulation Society recommend the use of GCS for prophylaxis among patients at an intermediate risk for VTE [1]. Anticoagulation or intermittent pneumatic compression (IPC) is recommended for patients at a high risk for VTE, such as those undergoing major orthopedic surgery, while anticoagulation plus IPC or GCS is recommended for patients at the highest risk for VTE [1]. However, the efficacy of the combined treatment of anticoagulation with GCS relative to anticoagulation alone for VTE prevention among patients undergoing major lower limb orthopedic surgery has not been evaluated. In this study, an exploratory analysis was performed to evaluate the efficacy of GCS combined with enoxaparin or edoxaban, a direct, oral, factor $\mathrm{Xa}$ inhibitor, using data from a phase 3 clinical study that assessed the efficacy and safety of edoxaban (vs enoxaparin) for the prevention of postoperative VTE in patients undergoing TKA.

\section{Methods}

\section{Study selection}

Three phase 3 studies involving patients undergoing TKA (TKA P3; STARS E-3 trial) [4], THA (THA P3; STARS J-5 trial) [5], or HFS (HFS P3; STARS J-4 trial) [6] were considered for inclusion in this analysis. A total of 716, 610, and 92 patients were enrolled in the TKA P3 [4], THA P3 [5], and HFS P3 [6] studies, respectively, of whom 594, 503, and 73, respectively, were evaluable for efficacy. These 3 studies were conducted between October 2008 and January 2010. In all 3 studies, the concomitant use of any method of mechanical prophylaxis, including GCS and IPC, was permitted. Table 1 summarizes the number of patients available for efficacy analysis stratified by the method of mechanical prophylaxis. Since there were few patients without mechanical prophylaxis in the THA P3 (1 patient) and HFS P3 (9 patients) studies, these 2 studies were excluded from the present analysis. In TKA P3, 129 patients received GCS
Table 1 Use of mechanical prophylaxis (efficacy analysis set)

\begin{tabular}{|c|c|c|c|c|}
\hline \multicolumn{2}{|c|}{ Mechanical prophylaxis } & \multirow{2}{*}{$\begin{array}{l}\text { TKA P3 } \\
N=594\end{array}$} & \multirow{2}{*}{$\begin{array}{l}\text { THA P3 } \\
N=503\end{array}$} & \multirow{2}{*}{$\begin{array}{l}\text { HFS P3 } \\
N=73\end{array}$} \\
\hline GCS & IPC & & & \\
\hline o & $x$ & 129 & 136 & 39 \\
\hline 0 & 0 & 238 & 274 & 15 \\
\hline$x$ & $\mathrm{O}$ & 123 & 92 & 10 \\
\hline$x$ & $x$ & 104 & 1 & 9 \\
\hline
\end{tabular}

GCS graduated compression stockings, HFS $P 3$ hip fracture surgery phase 3 study, IPC intermittent pneumatic compression, THA P3 total hip arthroplasty phase 3 study, TKA P3 total knee arthroplasty phase 3 study

$\mathrm{o}=$ patients receiving indicated treatment; $\mathrm{x}=$ patients without the indicated treatment

alone as mechanical prophylaxis and 104 patients had no mechanical prophylaxis. Data from the TKA P3 study were included in the present analysis.

\section{Study design and site selection}

TKA P3 was a randomized, double-blind, parallel-group study performed by the double-dummy method (Fig. 1) [4]. Edoxaban (30 mg) was administered orally once a day, while enoxaparin (20 mg) was injected subcutaneously twice a day for 11 to 14 days in accordance with standard practice. Edoxaban was administered 6 to $24 \mathrm{~h}$ after surgery, whereas enoxaparin treatment commenced 24 to $36 \mathrm{~h}$ following surgery. Venography was performed within $24 \mathrm{~h}$ after the last dose, and occurrence of VTE was determined by an independent Clinical Event Committee that was blinded to the treatment of the patients. The primary efficacy endpoint was the percentage of patients who developed asymptomatic DVT, symptomatic DVT, or symptomatic PE (the incidence of VTE).

In TKA P3, 716 patients were enrolled at 71 sites. To exclude possible bias due to selection of GCS depending on the severity of illness or the VTE risk of each patient, 18 sites where GCS had been used for selected patients were excluded from the present analysis. Thus, the remaining 422 patients from 53 sites were considered for inclusion in the present analysis. To avoid the influence of other mechanical methods (eg, IPC), patients who had also used IPC were excluded as well. Thus, the present analysis compared 101 patients who had used GCS alone as mechanical prophylaxis (53 from the edoxaban group and 48 from the enoxaparin group) with 100 patients without any mechanical prophylaxis (52 from the edoxaban group and 48 from the enoxaparin group), so a total of 201 patients were included in this assessment of the efficacy of GCS.

\section{Statistical analysis}

The incidence of VTE was summarized using descriptive statistics. Treatment comparisons were made using the $\chi 2$ test and the $95 \%$ confidence interval was calculated. 


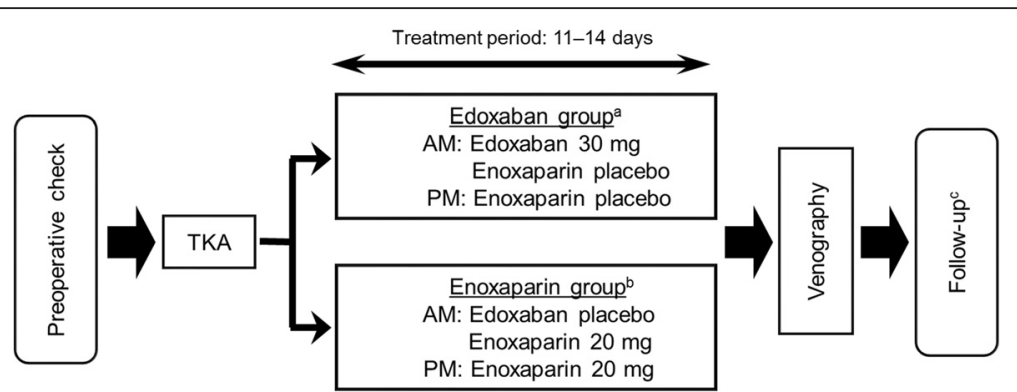

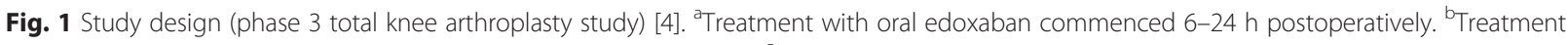
with subcutaneous enoxaparin commenced $24-36 \mathrm{~h}$ postoperatively. ${ }^{C}$ Follow-up occurred $25-35$ days after the last administered dose

\section{Results}

The demographics and baseline characteristics of patients included in this subanalysis are presented in Table 2. The mean age, body weight, and creatinine clearance were not different among patients regardless of anticoagulant and/or mechanical prophylaxis use.
Tourniquet use was lower, whereas the use of epidural and lumbar anesthesia was higher among patients receiving GCS relative to those without any mechanical prophylaxis. This difference was due to facility-specific practices; facilities employing GCS for prophylaxis had lower rates of tourniquet or general anesthesia use.

Table 2 Demographic and baseline characteristics summary

\begin{tabular}{|c|c|c|c|c|}
\hline \multirow[t]{3}{*}{ Demographic and other baseline characteristics } & \multicolumn{2}{|c|}{$\begin{array}{l}\text { No mechanical prophylaxis } \\
N=100\end{array}$} & \multicolumn{2}{|l|}{$\begin{array}{l}\text { GCS alone } \\
N=101\end{array}$} \\
\hline & Edoxaban & Enoxaparin & Edoxaban & Enoxaparin \\
\hline & $N=52$ & $N=48$ & $N=53$ & $N=48$ \\
\hline \multicolumn{5}{|l|}{ Sex, n (\%) } \\
\hline Male & $11(21.2)$ & $9(18.8)$ & $8(15.1)$ & $9(18.8)$ \\
\hline Female & $41(78.8)$ & $39(81.3)$ & $45(84.9)$ & 39 (81.3) \\
\hline \multicolumn{5}{|l|}{ Age, (y) } \\
\hline Mean \pm SD & $70.9 \pm 7.9$ & $71.7 \pm 7.8$ & $73.5 \pm 6.1$ & $73.2 \pm 7.0$ \\
\hline \multicolumn{5}{|l|}{ Weight, (kg) } \\
\hline Mean \pm SD & $63.2 \pm 10.2$ & $64.3 \pm 11.9$ & $56.8 \pm 10.2$ & $60.3 \pm 10.1$ \\
\hline \multicolumn{5}{|l|}{$\mathrm{CL}_{\mathrm{cr},}(\mathrm{mL} / \mathrm{min})$} \\
\hline Mean \pm SD & $78.3 \pm 24.6$ & $76.2 \pm 26.5$ & $71.1 \pm 20.1$ & $74.9 \pm 20.0$ \\
\hline \multicolumn{5}{|l|}{ Duration of surgery, (h:min) } \\
\hline Mean \pm SD & $2: 03 \pm 0: 44$ & $1: 59 \pm 0: 35$ & $1: 42 \pm 0: 26$ & $1: 45 \pm 0: 24$ \\
\hline Tourniquet use during surgery, n (\%) & $50(96.2)$ & $46(95.8)$ & $42(79.2)$ & $38(79.2)$ \\
\hline \multicolumn{5}{|l|}{ Method of anesthesia, n (\%) } \\
\hline General anesthesia & $43(82.7)$ & $35(72.9)$ & $24(45.3)$ & $20(41.7)$ \\
\hline Epidural anesthesia & $8(15.4)$ & $7(14.6)$ & $17(32.1)$ & $14(29.2)$ \\
\hline Lumbar anesthesia & $24(46.2)$ & $24(50.0)$ & $38(71.7)$ & $34(70.8)$ \\
\hline \multicolumn{5}{|c|}{ Duration from the end of surgery to the start of anticoagulation, (h:min) } \\
\hline Mean \pm SD & $20: 31 \pm 2: 39$ & $30: 41 \pm 3: 26$ & $19: 43 \pm 2: 59$ & $27: 08 \pm 2: 30$ \\
\hline \multicolumn{5}{|l|}{ Duration from surgery to ambulation (days) } \\
\hline Mean \pm SD & $2.2 \pm 0.7$ & $2.4 \pm 0.8$ & $3.6 \pm 1.4$ & $3.8 \pm 2.1$ \\
\hline \multicolumn{5}{|l|}{ Duration of treatment (days) } \\
\hline Mean \pm SD & $12.1 \pm 1.6$ & $11.6 \pm 2.6$ & $12.4 \pm 1.6$ & $12.4 \pm 1.7$ \\
\hline \multicolumn{5}{|l|}{ Duration of GCS use (days) } \\
\hline Mean \pm SD & - & - & $9.5 \pm 2.7$ & $9.6 \pm 2.7$ \\
\hline
\end{tabular}

$C L_{c r}$ creatinine clearance, GCS graduated compression stockings, $S D$ standard deviation 
Mean GCS use was 9.5 and 9.6 days for the edoxaban and enoxaparin groups, respectively. The mean (standard deviation) time interval between surgery and the start of postoperative anticoagulant therapy was $20 \mathrm{~h}$ and $7 \mathrm{~min}(2 \mathrm{~h}$ and $50 \mathrm{~min}$ ) for the edoxaban group and $28 \mathrm{~h}$ and $54 \mathrm{~min}$ ( $3 \mathrm{~h}$ and $29 \mathrm{~min}$ ) for the enoxaparin group. Enoxaparin was administered between 24 to $36 \mathrm{~h}$ after surgery, in accordance with enoxaparin prescribing practices [7], accounting for this difference in initiation of anticoagulation following surgery.

In the edoxaban group, the incidence of VTE was $3.8 \%$ $(2 / 53)$ and $5.8 \%$ (3/52) for patients with and without GCS mechanical prophylaxis, respectively ( $p=0.6312$; Table 3 ). In the enoxaparin group, the incidence of VTE was $8.4 \%$ $(4 / 48)$ for patients using GCS alone as mechanical prophylaxis, and $20.8 \%(10 / 48)$ for patients without mechanical prophylaxis $(p=0.0827)$. Combining the 2 groups, the incidence of VTE was $6.0 \%(6 / 101)$ and $13.0 \%(13 / 100)$ for patients with and without GCS mechanical prophylaxis. There were no deaths in this study. Of the 201 patients included in this analysis, no instances of symptomatic or asymptomatic PE and no instances of symptomatic DVT were observed; all patients had asymptomatic DVT.

\section{Discussion}

Anticoagulants prevent VTE by suppressing hypercoagulability [8], while GCS prevent VTE by increasing the venous blood flow velocity and impeding venous stasis in the legs [3]. Thus, 2 independent elements of Virchow's thrombogenic triad ("hypercoagulability" and "venous stasis") [8] are targeted when prophylaxis with anticoagulant therapy and GCS is combined. This is thought to be the mechanism by which combined therapy achieves a greater reduction in the incidence of VTE after major lower limb orthopedic surgery. Few studies have examined the efficacy of combined anticoagulant and GCS therapy relative to anticoagulation alone for VTE prevention among patients undergoing major orthopedic surgery [9]. A study involving patients undergoing THA in the UK revealed that the incidence of DVT or PE was not significantly different between patients receiving enoxaparin plus GCS compared to those receiving enoxaparin alone [10]. However, this study was limited by its small sample size, with 32 patients randomized to each treatment group [10]. Among Swedish patients undergoing THA, combined treatment with dextran (infused at anesthesia and for 2 days following surgery) and GCS was more effective for postoperative DVT prevention than treatment with dextran alone [11].

Although Japanese guidelines recommend the use of GCS with anticoagulation for prophylaxis among patients at the highest risk for VTE [1], the efficacy of this combined treatment relative to anticoagulation alone for Japanese patients undergoing major orthopedic surgery has previously not been reported. The findings from the present study suggest that concomitant treatment with anticoagulation and GCS resulted in a $>2$-fold reduction in VTE incidence relative to anticoagulation alone among Japanese patients undergoing TKA, although this difference was not statistically significant. Addition of GCS therapy led to a numerically greater reduction in VTE incidence primarily among patients receiving enoxaparin, while this reduction was less pronounced among those receiving edoxaban. This difference may have arisen partly because edoxaban was started earlier in the postoperative period; as a result, there was a smaller margin for further reduction of VTE occurrence with the addition of GCS among patients treated with edoxaban. Given the limited number of patients in this analysis $(n=201)$, and the delay in the initiation of anticoagulation with enoxaparin relative to edoxaban, these exploratory results need to be confirmed in larger, prospective, randomized trials.

\section{Conclusion}

To evaluate the efficacy of GCS for prevention of VTE, an exploratory analysis was performed by using data from a phase 3 clinical study of postoperative prophylaxis with edoxaban vs enoxaparin in patients undergoing TKA. The results of the analysis suggested that the occurrence of VTE was numerically lower among patients receiving anticoagulant plus GCS therapy relative

Table 3 Incidence of VTE ${ }^{a}$ in patients using GCS for prophylaxis

\begin{tabular}{|c|c|c|c|}
\hline & Edoxaban & Enoxaparin & Total \\
\hline & $N=105$ & $N=96$ & $N=201$ \\
\hline No mechanical prophylaxis & $5.8 \%(3 / 52)$ & $20.8 \%(10 / 48)$ & $13.0 \%(13 / 100)$ \\
\hline Mechanical prophylaxis with GCS alone & $3.8 \%(2 / 53)$ & $8.4 \%(4 / 48)$ & $6.0 \%(6 / 101)$ \\
\hline Chi-square test & $p=0.6312$ & $p=0.0827$ & $p=0.0872$ \\
\hline Difference between with and without GCS treatment groups & -2.0 & -12.5 & -7.1 \\
\hline $95 \% \mathrm{Cl}$ of the difference shown by the score test & $-12.2,7.8$ & $-26.8,1.9$ & $-15.7,1.2$ \\
\hline
\end{tabular}

Cl confidence interval, GCS graduated compression stockings, VTE venous thromboembolism

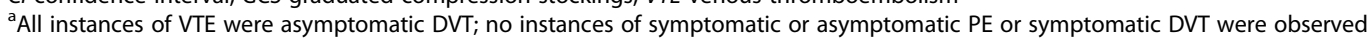


to those receiving anticoagulation alone, although this difference was not statistically significant. Further studies are required to confirm the findings of this preliminary analysis. Additionally, due to the limitations of this analysis, further studies need to be performed to evaluate the relative prophylactic efficacy of combined anticoagulant and GCS therapy compared with anticoagulation alone.

\section{Acknowledgements}

Medical writing and editorial support was provided by Sameera Kongara, PhD, of AlphaBioCom, LLC (King of Prussia, PA), which was sponsored by Daiichi Sankyo, Inc.

\section{Authors' contributions}

All authors were involved in the design of the study and performed the research. KA analyzed the data. All authors were involved in the development of the manuscript and provided approval for submission.

\section{Competing interests}

This study (ClinicalTrials.gov Identifier: NCT01181102) was sponsored by Daiichi Sankyo Co., Ltd (Tokyo, Japan). The study sponsors were involved in the design of the study and the collection and analysis of the data. TK, KI, and KA are employees of Daiichi Sankyo.

TF has been a consultant for Daiichi Sankyo Co., Ltd.; and Ono Pharmacy; received royalties from Century Medical and Showa Ikakogyo; and received compensation for speaker's bureau/paid presentations from Daiichi Sankyo Co., Ltd.; Century Medical; Ono Pharma; and Bayer. SF has been a consultant for Daiichi Sankyo Co., Ltd.; Astellas; and GlaxoSmithKline. ST has been a consultant for Daiichi Sankyo Co., Ltd., and GlaxoSmithKline. MN has been a consultant for Daiichi Sankyo Co., Ltd.

\section{Ethics approval and consent to participate}

All 3 studies considered for inclusion in this analysis were reviewed and approved by their respective institutional review boards [4-6]. These studies were conducted in compliance with the Declaration of Helsinki and Good Clinical Practice guidelines. Prior to enrollment, all patients participating in each of these studies provided written informed consent.

\section{Author details}

'Department of Orthopedic Surgery, Japan Community Healthcare Organization Osaka Hospital, 4-2-78 Fukushima, Fukushima-ku, Osaka 553-0003, Japan. ²Department of Orthopedic Surgery, Takarazuka Daiichi Hospital, 19-5 Kogetsu-cho, Takarazuka 665-0832, Japan. ${ }^{3}$ Daiichi Sankyo Co., Ltd., 3-5-1, Nihonbashi Honcho, Chuo-ku, Tokyo 103-8426, Japan. ${ }^{4}$ Clinical Data and Biostatistics Department, Daiichi Sankyo Co., Ltd., 1-2-58, Hiromachi, Shinagawa-ku, Tokyo 140-8710, Japan. ${ }^{5}$ Department of Orthopedic Surgery, Mishuku Hospital, 5-33-12 Shimomeguro, Meguro-ku, Tokyo 153-0051, Japan. ${ }^{6}$ Department of Cardiology and Nephrology, Mie University Graduate School of Medicine, 2-174 Edobashi, Tsu, Tsu 514-8507, Mie, Japan.

Received: 8 December 2015 Accepted: 25 May 2016

Published online: 08 June 2016

\section{References}

1. JCS Joint Working Group. Guidelines for the diagnosis, treatment and prevention of pulmonary thromboembolism and deep vein thrombosis (JCS 2009). Circ J. 2011:75(5):1258-81.

2. Anderson FA, Spencer FA. Risk factors for venous thromboembolism. Circulation. 2003;107(23 suppl 1):I-9-|-16.

3. Laryea J, Champagne B. Venous thromboembolism prophylaxis. Clin Colon Rectal Surg. 2013;26(3):153-9. doi:10.1055/s-0033-1351130.

4. Fuji T, Wang CJ, Fujita S, Kawai Y, Nakamura M, Kimura T, et al. Safety and efficacy of edoxaban, an oral factor Xa inhibitor, versus enoxaparin for thromboprophylaxis after total knee arthroplasty: the STARS E-3 trial. Thromb Res. 2014;134(6):1198-204. doi:10.1016/j.thromres.2014.09.011.

5. Fuji T, Fujita S, Kawai Y, Nakamura M, Kimura T, Fukuzawa M, et al. Efficacy and safety of edoxaban versus enoxaparin for the prevention of venous thromboembolism following total hip arthroplasty: STARS J-V. Thromb J. 2015;13:27. doi:10.1186/s12959-015-0057-x.
6. Fuji T, Fujita S, Kawai Y, Nakamura M, Kimura T, Kiuchi Y, et al. Safety and efficacy of edoxaban in patients undergoing hip fracture surgery. Thromb Res. 2014;133(6):1016-22. doi:10.1016/j.thromres.2014.03.009.

7. Clexane ${ }^{\circledast}$ for Subcutaneous Injection Kit 2000 IU. Tokyo, Japan: Sanofi-Aventis K.K; 2012 [Package Insert (Ver. 8), in Japanese].

8. Turpie AG, Esmon C. Venous and arterial thrombosis-pathogenesis and the rationale for anticoagulation. Thromb Haemost. 2011;105(4):586.

9. Sachdeva A, Dalton M, Amaragiri SV, Lees T. Elastic compression stockings for prevention of deep vein thrombosis. Cochrane Database Syst Rev. 2010; 7:CD001484. doi:10.1002/14651858.CD001484.pub2.

10. Kalodiki EP, Hoppensteadt DA, Nicolaides AN, Fareed J, Gill K, Regan F, et al. Deep venous thrombosis prophylaxis with low molecular weight heparin and elastic compression in patients having total hip replacement. A randomised controlled trial. Int Angiol. 1996;15(2):162-8.

11. Ohlund C, Fransson SG, Starck SA. Calf compression for prevention of thromboembolism following hip surgery. Acta Orthop Scand. 1983;54(6):896-9.

\section{Submit your next manuscript to BioMed Central and we will help you at every step:}

- We accept pre-submission inquiries

- Our selector tool helps you to find the most relevant journal

- We provide round the clock customer support

- Convenient online submission

- Thorough peer review

- Inclusion in PubMed and all major indexing services

- Maximum visibility for your research

Submit your manuscript at www.biomedcentral.com/submit
C Biomed Central 